\title{
Reflective practice groups and nurse professional quality of life
}

AUTHORS

\author{
MADISON KM SUNDGREN ${ }^{1}$ \\ BA Psychology (Honours) \\ PRUDENCE M MILLEAR ${ }^{1}$ PhD, Ba Psychology \\ (Honours)
}

CHRIS DAWBER ${ }^{2}$ Ma MH Nursing, Ma Psychotherapy,

Cert Dev Psych, MHN Cred

LUIGI MEDORO² DClinPsych, PostGrad Dip Psych, BA
1 School of Health and Behavioural Sciences, University of the Sunshine Coast, Queensland, Australia

2 Mental Health and Addiction Services, Sunshine Coast Hospital and Health Service, Queensland, Australia

\section{CORRESPONDING AUTHOR}

CHRIS DAWBER Sunshine Coast University Hospital \& Health Service, c/o PO Box 367, Golden Beach, QLD, Australia 4551. Email: Chris.Dawber@health.qld.gov.au

\section{ABSTRACT}

Objective: This study aimed to examine the relationship between the quality of reflective practice groups and nurses' professional quality of life.

Background: Previous nursing research has indicated that reflective practice groups are positively associated with personal resources, job resources, and professional quality of life for nurses. The specific impact of the groups however, has not been distinguished from the impact of personal and job resources, and the explanatory mechanisms for the associations are unclear.

Design/Methods: This study utilised a crosssectional, quantitative research methodology with 184 Australian nurses from a regional teaching hospital who attended reflective practice groups (88.5\% female). Surveys captured demographics, personal resources (i.e. self-efficacy), job resources (i.e. job autonomy, skill discretion, job social support, and group cohesion) and perceived quality of reflective practice groups, as measured by the Clinical Supervision Evaluation Questionnaire, alongside professional quality of life outcomes.

Results: Participants who rated reflective practice groups as 'high quality' or more effective were associated with significantly higher scores for personal and job resources of self-efficacy, autonomy, skill discretion, social support, and group cohesion. These resources, in turn, correlated with more positive professional quality of life scores; in particular compassion satisfaction and burnout.

Discussion: While the study used cross-sectional data and causality cannot be inferred, the findings do indicate a clear association between attending effectively facilitated reflective practice groups and greater personal and job resources. This may indicate that the groups provide nurses with an opportunity to build or enhance these resources. Furthermore, it is proposed that this could explain a mechanism by which reflective practice groups can indirectly impact positively on professional quality of life for nurses by building resources. Future research should explore causality longitudinally.

Conclusion: This study provides evidence that nurses who perceive the groups favorably have greater social support, enhanced self-efficacy, more autonomy, and increased skill discretion. These factors also align with the restorative and formative benefits of supervision. Greater personal and job resources, in turn, can enhance professional quality of life. Whilst mediating pathways have been identified, it is still not possible to definitively attribute the development of these resources to group participation. 
Implications for Research, Policy, and Practice: There is growing evidence to support reflective practice groups as a viable form of group clinical supervision for nurses. It is important to ensure reflective practice groups are effectively facilitated.

\section{What is already known about the topic?}

Correlational data indicates that high quality reflective practice groups may offer a cost-effective form of group clinical supervision, for nurses; providing support and helping them deal with what can be a challenging professional environment. Reflective practice groups have been linked to a range of benefits across individual and organisational levels, including; 1 ) increased job satisfaction, 2) higher quality of patient care, less critical incidents, 3 ) higher rates of staff retainment and reduced leave, and 4) enhancing the integrity of the nursing profession through accountability and skill development.

\section{What this paper adds}

Despite these claims, little is known regarding the mechanisms underpinning these documented benefits. This study provides a theoretically driven explanation; that reflective practice groups provide an opportunity for job crafting, in which nurses actively build resources (i.e. skills development critical thinking and social support), mitigating some of the negative psychological consequences associated with nursing. This information can help guide policy, practice, and the development of effective interventions to provide support and supervision for nurses.

Keywords: Nursing, group supervision, reflective practice, compassion satisfaction, burnout, stress.

\section{INTRODUCTION}

Nursing can be a rewarding profession, but it can also be stressful. Repeated exposure to human suffering and confounding contextual factors can contribute to a reduction in nurses professional quality of life (ProQoL). ${ }^{1,2}$ Low ProQoL within nursing can contribute to increased absenteeism and staff turnover ${ }^{3,4}$ It is anticipated that given the combination of poor retention rates and an ageing workforce, Australia will face a critical nursing shortage; with many nurses now opting for part-time employment. ${ }^{5}$ To contextualise, it is expected by 2030 there will be a shortfall of approximately 123,000 Australian nurses, $31 \%$ less than required. ${ }^{6}$ Developing interventions that can have a positive influence on nursing ProQoL may be one way of meeting this challenge.

\section{BACKGROUND}

The construct of ProQoL consists of burnout, secondary traumatic stress, and compassion satisfaction. ${ }^{2}$ Burnout has been conceptualised as a negative psychological reaction to prolonged chronic stress, characterised by physical, emotional, and mental exhaustion., ${ }^{2,7}$ Secondary traumatic stress occurs when professionals are traumatised after vicariously adopting patient trauma. Burnout and secondary traumatic stress collectively termed as compassion fatigue, encapsulating the negative characteristics of providing care. ${ }^{2}$ Compassion satisfaction refers to the positive characteristics, defined as the gratification one derives from their caring role. ${ }^{2}$
The job demands-resources model, ${ }^{8}$ provides a framework for understanding how to increase ProQoL. The primary assumption of the job demands-resources model is that employee wellbeing is influenced by specific job characteristics, such as demands and resources. Resources refer to aspects of a job that promote wellbeing through fostering personal growth, enabling employees to achieve goals, or by reducing demands. Resources may occur at a personal level (e.g. self-efficacy) or an occupational level (e.g. managerial support). In contrast, job demands refer to aspects of a job that are associated with costs, as they require sustained effort (physical, emotional, or cognitive) to overcome. ${ }^{9}$ Nursing studies using cross-sectional correlational data, ${ }^{10}$ and longitudinal evidence, ${ }^{11}$ have demonstrated excessive demands predict health impairment processes which increase the risk of burnout. In contrast, job resources have been associated with motivational processes that stimulate work engagement and protect against the negative impact of demands. ${ }^{12}$ Given that nurses are faced with a difficult combination of low resources and high demands, ${ }^{13}$ it is essential to investigate ways to increase nursing resources to buffer against high workplace demands, and subsequently, increase ProQoL. ${ }^{1}$

Within the nursing field, it is proposed that reflective practice groups (RPGs), a form of group clinical supervision, may provide an opportunity for nurses to increase job resources. ${ }^{1}$ RPGs have been presented as both a form of group clinical supervision, ${ }^{14-17}$ and a resource. ${ }^{1}$ Recent theoretical extensions have included job crafting within the job demands-resources model. ${ }^{18}$ Job crafting is defined as a process in which workers independently modify job 
demands and resources. ${ }^{19}$ By proactively shaping work to suit their own needs, abilities, and preferences, employees experience more satisfaction and work engagement. For instance, Tims and colleagues, ${ }^{20}$ found job crafting increased resources (i.e. autonomy, task variety, social support, feedback) in a sample of chemical engineers, revealing a significant increase in wellbeing; lower burnout, increased job satisfaction, and work engagement. Job crafting has been defined as a self-initiated work design process involving proactive strategies to change characteristics of a job in a way that aligns more closely with personal needs, goals, and skills. ${ }^{18}$

It is reasonable to consider RPGs as a job crafting exercise, given that the model defines the groups as voluntary, collaborative and nurse-driven, and with the aim of mitigating stress, increasing skills and supporting nurses with the interpersonal aspects of their work. ${ }^{14}$ RPGs explore a range of clinical, professional, ethical and organisational issues within a supportive group setting, ${ }^{14,21}$ with a particular focus on the personal and interpersonal aspects of nursing care. ${ }^{17}$ Critical reflection is prompted through facilitator interventions directed towards 'the group'; the aim being to access a range of experiences, reactions and perspectives in order to enrichen the reflective experience. ${ }^{14,17,21}$ It is reasonable to consider RPGs as a job crafting exercise, given that they are voluntary, nurse-driven and aim to increase skills and support nurses. ${ }^{14}$ Nurses have the freedom to direct RPGs in order to align with their needs, however, it is argued that for nurses to proactively foster resources, they need to perceive RPGs as trustworthy and constructive. ${ }^{16}$

Dawber, ${ }^{14}$ specified several strategies to promote the delivery of high-quality RPGs within a clinical healthcare setting. For instance, to enhance RPG effectiveness the unique culture of each clinical area and specific requirements and demands was acknowledged. Dawber, ${ }^{14}$ also allowed the group to decide the frequency and duration of RPGs and considered workplace dynamics to ensure facilitators enabled the group to address contextual factors (e.g. conflict, unsupportive organisational culture). Furthermore, facilitators must foster psychological safety by embedding principles of confidentiality, openness, mutual respect, and acceptance of diversity throughout RPG discussions. It is advised that conversations during RPG are semi-structured and collaborative, with the facilitator addressing the 'group' rather than individuals to reduce pressure to participate and promote non-threatening communication. ${ }^{14}$ Additionally, a 12-month pilot study indicated a high level of nurse satisfaction with the quality of their RPGs, ${ }^{22}$ as measured by the Clinical Supervision Evaluation Questionnaire. ${ }^{21}$ Overall, it appears that the RPG model proposed by Dawber, ${ }^{14}$ parallels many core elements of a job crafting experience. That is, nurses are deemed active agents in aligning RPGs to fulfill their needs.
In line with job crafting principles, consistent with the wider literature, and in keeping with the job demandsresources model, RPG benefits are proposed to stem from the collaborative nature of group interactions; such as gaining social support, cohesion, and connection with peers through sharing and reflecting on clinical information. ${ }^{14}$ Higher quality clinical supervision groups have been associated with positive psychological outcomes in cross-sectional correlational research. For instance, higher quality group supervision increased job satisfaction in Finnish mental health nurses, ${ }^{23}$ and Danish hospital nurses $(N=136)$, reported more frequent use of rational coping, greater job satisfaction, vitality, and significantly lower burnout and stress. ${ }^{24}$ For Finnish hospital nurses ( $N=148$ ), higher quality group clinical supervision increased job resources (e.g. autonomy, social support) and organisational commitment and lowered burnout. ${ }^{25}$ Taken together, this research suggests that attending quality RPGs increase job resources and enhance nurses wellbeing and ProQoL. It is likely that effective group clinical supervision will promote work satisfaction and provide protection from emotional and physical exhaustion.

From a theoretical perspective, it is argued that RPGs provide nurses with opportunities to actively foster job resources to manage their demands, mitigate burnout, and improve ProQoL. However, the mechanisms explaining how RPGs might bring about change are still being empirically tested and therefore, still poorly understood. Despite the lack of quantitative evidence, qualitative studies allude to the benefits of RPGs being consistent with the job demandsresources model. ${ }^{26,27}$

The aims of the present study were to extend previous research by more closely examining the relationship between RPGs and ProQoL in the context of the job demandsresources model; assessing the extent that personal and job resources might mediate (explain) the relationship between more effective RPGs and greater ProQoL. The first aim was to show whether nurses who rated RPGs as higher quality would have significantly higher personal and job resources (i.e. greater levels of self-efficacy, autonomy, skill discretion, job social support, and group cohesion). The second aim was to show how these resources mediate the relationship between more effective RPGs and greater ProQoL, as measured by significantly lower burnout, secondary traumatic stress, and greater compassion satisfaction.

\section{METHODS}

\section{DESIGN}

Cross-sectional data was collected as part of a larger longitudinal quasi-experimental post-design project, titled 'Evaluating Reflective Practices for Nursing Staff in an Acute Hospital Setting'. 


\section{PARTICIPANTS}

Registered nurses ( $N=184,88.5 \%$ female) were recruited from hospital wards with RPGs at an Australian public tertiary hospital. Participant demographic and professional characteristics are presented in Table 1 . Information regarding RPG participation is presented in Table 2.

TABLE 1: NURSES SOCIODEMOGRAPHIC AND PROFESSIONAL CHARACTERISTICS $(N=184)$

\begin{tabular}{|l|r|r|}
\hline Variable & $\mathbf{n}$ \\
\hline Gender & 21 & 11.50 \\
\hline Male & 162 & 88.50 \\
\hline Female & \multicolumn{3}{|r|}{} \\
\hline Nursing speciality & 23 & 12.50 \\
\hline Mental Health & 85 & 46.20 \\
\hline Medical & 20 & 10.87 \\
\hline Surgical & 56 & 30.43 \\
\hline Other Nursing Area & 14.48 & \\
\hline Mean years nursing experience (SD) & $(11.82)$ & \\
\hline Mean hours of work per week (SD) & 31.96 & \\
\hline
\end{tabular}

a Missing unreported data, $n=183$. SD, standard deviation.

TABLE 2: SUMMARY OF DESCRIPTIVE STATISTICS OF REFLECTIVE PRACTICE GROUP ATTENDANCE, FREQUENCY OF ATTENDANCE, AND PREFERENCE FOR ATTENDANCE $(N=184)$

\begin{tabular}{|c|c|c|}
\hline Variable & Frequency & Percent \\
\hline \multicolumn{3}{|l|}{ Attendance } \\
\hline $1-6$ & 102 & 55.4 \\
\hline $7-12$ & 32 & 17.4 \\
\hline $13-18$ & 15 & 8.2 \\
\hline $19-24$ & 10 & 5.4 \\
\hline $25-32$ & 6 & 3.3 \\
\hline $33+$ & 19 & 10.3 \\
\hline \multicolumn{3}{|l|}{ Frequency of attendance ${ }^{a}$} \\
\hline Never & 2 & 1.1 \\
\hline Just once & 25 & 13.7 \\
\hline Once a year & 10 & 5.5 \\
\hline Twice a year & 34 & 18.7 \\
\hline Every three months & 35 & 19.2 \\
\hline Every two months & 28 & 15.4 \\
\hline Every month & 48 & 26.4 \\
\hline \multicolumn{3}{|c|}{ Preferred frequency of attendance ${ }^{a}$} \\
\hline Never & 2 & 1.1 \\
\hline When it is convenient & 42 & 23.1 \\
\hline Just when I need it & 21 & 11.5 \\
\hline As often as the groups run & 117 & 64.3 \\
\hline
\end{tabular}

a Missing unreported data, $n=182$.

\section{INTERVENTION}

RPGs were delivered using the model proposed by Dawber. ${ }^{14}$ This model of RPG is process-focused, semi-structured in nature, and group-led which enables nurses to shape the groups to their needs (e.g. duration, frequency, clinical discussions). Facilitators support nurses to reflect on their practice by providing psychological safety through containment and guidance (i.e. identifying resistance, prompting reflection through gentle questioning and open curiotsity). Facilitators had undergone a six to 12-month training program to deliver RPGs in this model. ${ }^{14}$ Nursing staff attended RPGs voluntarily, with RPGs being offered within a designated and protected education time. RPGs were delivered in a confidential space, adjacent to clinical work environments. RPGs were typically delivered as 6o-minute sessions, on a fortnightly to monthly basis. Attendance was supported by hospital management; however, managers did not attend.

\section{PROCEDURE}

Participants were recruited at the beginning of RPG sessions. Participants were provided with information and consent forms outlining study aims and non-identifiable nature of data, questionnaire, pens, and sealable envelopes to return documentation confidentially. Facilitators and researchers left the room whilst questionnaires were completed. Participants were advised that the data would be used to evaluate the impact of RPGs and its influence on the wider discipline of nursing.

Ethical approval (HREC/18/QPCH/132) was granted by both the hospital and university ethics committees, and informed consent was obtained prior to participation. Participants returned completed consent forms and questionnaires in sealed envelopes. Documents were separated and treated confidentially.

\section{MEASURES}

Demographic information included gender, age, weekly working hours, nursing department, and years working in nursing. All instruments demonstrated acceptable psychometric properties for use with nursing populations.

Reflective practice group quality. Nurse perception of RPG quality was measured using the 14 -item Clinical Supervision Evaluation Questionnaire (CSEQ). ${ }^{21}$ Participants were prompted to reflect on their previous experiences attending RPGs and respond by indicating their agreement to each item. Items were rated from (-2) strongly disagree to (2) strongly agree. Higher scores indicated higher perceptions of RPG effectiveness. The CSEQ was developed to assess three overarching components of group based clinical supervision groups, the purpose, process, and impact of the RPGs, as theorised by Proctor and colleagues. ${ }^{28}$ That is, do nurses perceive RPGs as meeting their own needs and 
needs of the service (purpose), experience RPGs as providing support (process), and lastly, perceive RPGs as facilitating positive change (impact). ${ }^{21,28}$ Items for the CSEQ were generated through input from group facilitators delivering clinical supervision and through literature focused on evaluating clinical supervision groups. Construct validity was demonstrated through scores on the CSEQ positively correlating with expected outcome measures, such as general measure of staff perception of clinical supervision. ${ }^{21}$ The CSEQ was chosen as it was developed to evaluate group clinical supervision, which was aligned with the "nonmanagerial peer group" style supervision offered within the current study. ${ }^{14}$ The CSEQ demonstrated reliability within the current study $(\alpha=.93)$ which is consistent with previous research. ${ }^{29}$ Scores for the CSEQ are summed, with higher scores indicating greater perception of RPG effectiveness. Scores range from -16 to 24 , with scores greater than 14 indicating a positive perception. Based on mean scores on the CSEQ, nurses within the current study appeared to perceive RPGs as positive intervention (see Table 3).

Self-efficacy. Self-efficacy was measured using the 10-item Generalised Self-Efficacy Scale. ${ }^{30}$ Items were rated from (1) not at all true to (4) exactly true. Items were summed, with total scores ranging from 10 to 40 . Higher scores reflected greater levels of self-efficacy. This scale demonstrated good internal consistency within the current study $(\alpha=.85)$.

Job autonomy. Autonomy was measured using the four-item Job Autonomy Scale. ${ }^{31}$ Items were rated from (1) never to (5) always. Items were totalled, ranged between 4 and 20, and higher scores indicated greater levels of autonomy. Cronbach's alpha within the current study was .85 .

Skill discretion. Job skill discretion was measured using the sixitem Skill Discretion Scale. ${ }^{32}$ Items were rated from (1) strongly disagree to (5) strongly agree. Items were summed, with scores ranging between 6 to 30, with higher scores indicate greater skill discretion. This scale demonstrated acceptable internal consistency $(\alpha=.60)$.

Job social support. Job social support was measured using the four-item Job Social Support Scale. ${ }^{33}$ Items were rated from, (1) strongly disagree to (5) strongly agree. Items were summed, with scores ranging from 4 to 20 , with higher scores indicating greater job social support. This scale demonstrated good internal consistency within the current sample ( $\alpha=$.79).

Group cohesion. Group cohesion was measured using the seven-item Group Cohesiveness Scale. ${ }^{34}$ Items were rated from (1) strongly disagree to (5) strongly agree. Cronbach's alpha within the current study was good $(\alpha=.86)$.

Professional quality of life. Professional quality of life was measured with the 30 -item ProQoL-5 scale, ${ }^{2}$ divided into three 10-item subscales: Burnout, 'I feel trapped in my job as a nurse' $(\alpha=.77)$; Secondary Traumatic Stress, 'I feel as though I am experiencing the trauma of someone I have cared for' $(\alpha=.81)$; and Compassion Satisfaction, 'I believe I can make a difference through my work' $(\alpha=.84)$. Items were rated from (1) never to (5) very often. Scores for each subscale were summed and converted to $t$-scores, with a mean of 50 and standard deviation of 10. Higher scores on Compassion Satisfaction and low scores on Burnout and Secondary Traumatic Stress suggest better professional health. Percentile cut offs are provided for low, moderate, and high levels of each component of professional quality of life. ${ }^{2}$

\section{DATA ANALYSIS}

Analyses were conducted with the Statistical Package for the Social Sciences (SPSS; Version 24.0) program. To verify the mediating effect of personal and job resources (i.e. self-efficacy, autonomy, skill discretion, social support, and group cohesion) on the relationship between greater RPG quality and greater ProQoL (as measured by lower burnout, lower secondary traumatic stress, and greater compassion satisfaction), three separate parallel mediation regression analyses were conducted using the PROCESS macros (Model 4), Version 3.o for SPSS. 35 All assumptions of multiple regression were met. There was no assumed causal ordering or reverse causality effects between examined variables.

Mediation regression examines the relationship between $X$ and $Y$, where $X$ is the predictor variable, $M_{j}$ refers to mediator variables that transmit the causal effect of $X$ to $Y$, and $Y$ is the outcome. ${ }^{35}$ The conceptual model of the hypothesised relationship is shown in Figure 1. In parallel mediation regression analysis, blocks of variables are examined to determine whether mediation has occurred. Blocks include; total effect, direct effect, and indirect effect. The total effect (c) represents a combination of a direct effect ( $\left.c^{\prime}\right)$ of $X$ on $Y$ and indirect effect $(a b)$ of $X$ on $Y$, which is transmitted through $M$. That is, the total effect between $X$ and $Y$ is comprised of a direct relationship and an indirect relationship. As shown in Figure 1, pathways are labelled as follows: ' $a$ ' pathway $(X \rightarrow M)$, ' $b$ ' pathway $(M \rightarrow Y)$, and the specific indirect effect 'ab' pathway $(X \rightarrow M \rightarrow Y)$.

CSEQ scores measuring the effectiveness of RPGs, were entered across all models as the predictor variable $(X)$. Self-efficacy $\left(M_{1}\right)$, autonomy $\left(M_{2}\right)$, skill discretion $\left(M_{3}\right)$, social support $\left(M_{4}\right)$, and group cohesion $\left(M_{5}\right)$ were entered simultaneously (i.e. parallel mediators) for all analyses. The three outcomes ( $Y$ ) were Burnout, Secondary Traumatic Stress, and Compassion Satisfaction. Bias-corrected confidence intervals were calculated using 10,00o bootstrap samples. Mediation is significant if the confidence interval of the specific indirect effect excludes zero. The sample size of 184 was deemed acceptable in accordance with Fritz and Mackinnon's, ${ }^{36}$ empirical estimates of sample size needed for .8o power $(\alpha=.05)$. 
a) Direct Pathway

\begin{tabular}{|c|c|c|}
\hline \begin{tabular}{c} 
Effective \\
Reflective Practice Groups \\
\cline { 2 - 2 }
\end{tabular} & $c \longrightarrow \begin{array}{c}\text { Professional Quality of Life } \\
(X)\end{array}$ \\
\hline
\end{tabular}

b) Indirect or Mediated Pathway

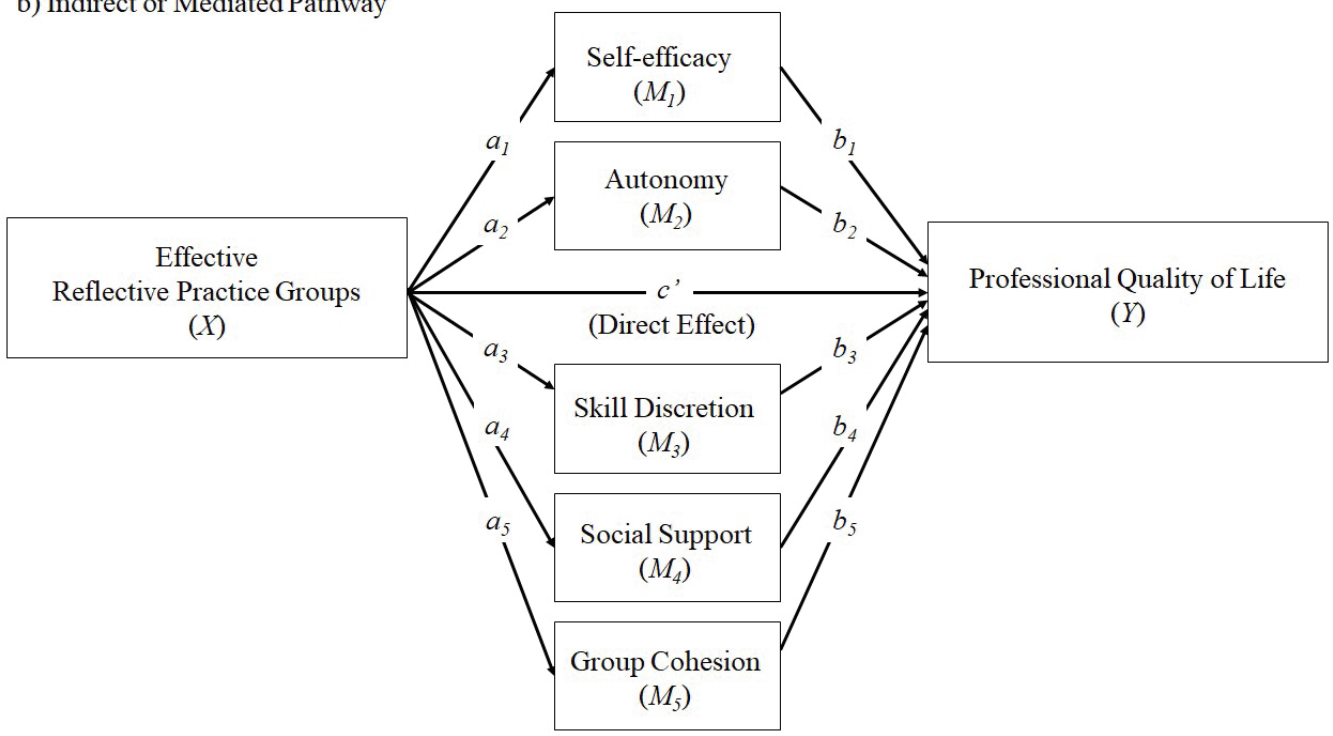

FIGURE 1: CONCEPTUAL DIAGRAM OF A PARALLEL MULTIPLE MEDIATION MODEL TESTING THE RELATIONSHIP BETWEEN THE EFFECTIVENESS OF RPGS, AS MEASURED BY CSEQ AND PROQOL (AS MEASURED BY BURNOUT, SECONDARY TRAUMATIC STRESS, AND COMPASSION SATISFACTION). PATHWAYS HAVE BEEN NUMBERED 1 TO 5 BASED ON THE CORRESPONDING PROPOSED MEDIATOR VARIABLE.

\section{RESULTS}

Mean scores, standard deviations, and correlations between study variables are presented in Table 3. Participants who perceived RPGs as more effective reported significantly lower levels of burnout and significantly higher levels of self-efficacy, autonomy, skill discretion, social support, group cohesion, and compassion satisfaction. Unexpectedly, the correlation between RPG quality and secondary traumatic stress was insignificant and in the positive direction.

TABLE 3: MEANS, STANDARD DEVIATIONS, AND ZERO-ORDER CORRELATIONS BETWEEN REFLECTIVE PRACTICE GROUP QUALITY, SELF-EFFICACY, AUTONOMY, SKILL DISCRETION, SOCIAL SUPPORT, GROUP COHESION, BURNOUT, SECONDARY TRAUMATIC STRESS, AND COMPASSION SATISFACTION $(N=184)$

\begin{tabular}{|c|c|c|c|c|c|c|c|c|c|c|c|}
\hline Variable & M & (SD) & 1 & 2 & 3 & 4 & 5 & 6 & 7 & 8 & 9 \\
\hline 1. RPG Quality & 15.70 & $(7.43)$ & - & & & & & & & & \\
\hline 2. Self-efficacy & 31.20 & (3.41) & $.33^{\star \star}$ & - & & & & & & & \\
\hline 3. Autonomy & 13.49 & $(2.65)$ & $.23^{\star *}$ & $.32^{\star \star}$ & - & & & & & & \\
\hline 4. Skill Discretion & 24.23 & $(2.58)$ & $.39^{\star \star}$ & $.26^{\star \star}$ & $.21^{\star \star}$ & - & & & & & \\
\hline 5. Social Support & 16.16 & $(2.53)$ & $.18^{*}$ & $.18^{*}$ & $.36^{\star *}$ & $.25^{\star \star}$ & - & & & & \\
\hline 6. Group Cohesion & 27.35 & $(3.51)$ & $.31^{\star *}$ & $.25^{\star \star}$ & $.32^{\star \star}$ & $.26^{\star \star}$ & $.49^{\star \star}$ & - & & & \\
\hline 7. Burnout & 50.38 & (9.94) & $-.18^{\star}$ & $-.37^{\star \star}$ & $-.45^{\star \star}$ & $-.22^{\star \star}$ & $-.36^{\star \star}$ & $-.26^{\star \star}$ & - & & \\
\hline 8. Secondary Traumatic Stress & 50.10 & $(9.98)$ & .09 & $-.21^{\star \star}$ & $-.26^{\star \star}$ & -.08 & $-.27^{\star \star}$ & -.03 & $.57^{\star \star}$ & - & \\
\hline 9. Compassion Satisfaction & 49.71 & $(10.15)$ & $.23^{\star \star}$ & $.32^{\star \star}$ & $.43^{\star \star}$ & $.35^{\star \star}$ & $.38^{\star \star}$ & $.30 \star \star$ & $-.73^{\star \star}$ & $-.29 * \star$ & - \\
\hline
\end{tabular}

M, Means; SD, Standard Deviation; RPG Quality, Reflective Practice Group Quality as measured by scores on Clinical Supervision Evaluation Questionnaire. ${ }^{\star} p<.05 ;{ }^{\star \star} p<.01 ;{ }^{\star \star \star} p<.001$. 
RELATIONSHIP BETWEEN RPG QUALITY AND RESOURCES

TABLE 4: THE STANDARDISED INDIRECT EFFECTS FOR THE RELATIONSHIP BETWEEN EFFECTIVE REFLECTIVE PRACTICE GROUPS AND PROFESSIONAL QUALITY OF LIFE THROUGH THE MEDIATORS OF SELF-EFFICACY, AUTONOMY, SKILL DISCRETION, SOCIAL SUPPORT AND GROUP COHESION

\begin{tabular}{|c|c|c|c|c|c|c|c|c|c|}
\hline \multirow[t]{3}{*}{$x$} & \multirow[t]{3}{*}{$M$} & \multirow[t]{3}{*}{$\mathrm{Y}$} & \multirow{2}{*}{\multicolumn{2}{|c|}{$\begin{array}{c}\text { Total Effect } \\
\text { (c) }\end{array}$}} & \multirow{2}{*}{\multicolumn{2}{|c|}{$\begin{array}{c}\text { Direct Effect } \\
\left(c^{\prime}\right)\end{array}$}} & \multicolumn{3}{|c|}{ Indirect Effect } \\
\hline & & & & & & & \multirow{2}{*}{$\begin{array}{r}(a \times b) \\
\beta\end{array}$} & \multicolumn{2}{|c|}{$95 \% \mathrm{Cl}$} \\
\hline & & & B & (SE) & B & (SE) & & LL & UL \\
\hline \multirow[t]{5}{*}{ RPGs } & Self-efficacy & $\mathrm{BO}$ & $-0.23^{\star}$ & $(0.10)$ & 0.03 & $(0.96)$ & $-.08^{\star}$ & -0.13 & -0.03 \\
\hline & Autonomy & & & & & & $-.07^{\star}$ & -0.12 & -0.02 \\
\hline & Skill Discretion & & & & & & -.02 & -0.08 & 0.03 \\
\hline & Social Support & & & & & & $-.04^{*}$ & -0.08 & -0.001 \\
\hline & Group Cohesion & & & & & & -.001 & -0.05 & 0.05 \\
\hline \multirow[t]{5}{*}{ RPGs } & Self-efficacy & STS & 0.18 & $(0.10)$ & $0.29^{* *}$ & $(0.10)$ & $-.06^{\star}$ & -0.12 & -0.01 \\
\hline & Autonomy & & & & & & $-.04^{\star}$ & -0.09 & -0.01 \\
\hline & Skill Discretion & & & & & & -.02 & -0.09 & 0.04 \\
\hline & Social Support & & & & & & $-.05^{\star}$ & -0.10 & -0.01 \\
\hline & Group Cohesion & & & & & & $.05^{\star}$ & 0.01 & 0.10 \\
\hline \multirow[t]{5}{*}{ RPGs } & Self-efficacy & CS & $0.31^{\star \star}$ & $(0.10)$ & -0.01 & $(0.10)$ & .04 & -0.003 & 0.10 \\
\hline & Autonomy & & & & & & $.06^{*}$ & 0.02 & 0.10 \\
\hline & Skill Discretion & & & & & & $.08^{*}$ & 0.02 & 0.15 \\
\hline & Social Support & & & & & & $.04^{*}$ & 0.002 & 0.08 \\
\hline & Group Cohesion & & & & & & .01 & -0.04 & 0.06 \\
\hline
\end{tabular}

$N=184 . X$, predictor variable; $M$, mediating variable; $Y$, outcome variable; RPGs, reflective practice group effectiveness, as measured by scores on Clinical Supervision Evaluation Questionnaire; BO, Burnout, STS, Secondary Traumatic Stress, CS, Compassion Satisfaction; Cl, confidence interval; $\mathrm{LL}$, lower limit; UL, upper limit. Significant findings are in boldface. ${ }^{\star} p<.05 ;{ }^{\star \star} p<.01 ;{ }^{\star \star \star} p<.001$.

First, the $a$ paths were examined. That is, the relationship between higher quality RPGs (i.e. higher scores on CSEQ) and each mediator (i.e. self-efficacy, autonomy, skill discretion, social support, and group cohesion). Standardised coefficients are presented in Figures 2 to 4 and reveal significant positive relationships between CSEQ scores and all proposed resources. That is, higher quality RPGs predicted significantly greater self-efficacy $\left(R^{2}=.11, F(1,182)=21.57, p<.001\right)$, autonomy $\left(R^{2}=.05, F(1,182)=9.79, p<.01\right)$, skill discretion $\left(R^{2}=.15\right.$, $F(1,182)=32.48, p<.001)$, social support $\left(R^{2}=.03, F(1,182)=6.33\right.$, $p<.05)$, and group cohesion $\left(R^{2}=.09, F(1,182)=18.71, p<.001\right)$. This suggests attending more effective (high quality) RPGs fosters self-efficacy, autonomy, skill discretion, social support, and group cohesion.

\section{THE MEDIATING ROLE OF PERSONAL AND JOB RESOURCES}

To assess whether the relationship between higher quality RPGs and greater ProQoL was mediated (explained) by the mediators, the following were examined for each component of ProQoL; total effect (c), direct effect ( $\left.c^{\prime}\right)$, effect of mediators $(b)$, and lastly, specific indirect effects $(a b)$.
Burnout. The standardised coefficients of pathways between RPG quality and Burnout scores are presented in Figure 2. As shown in Table 4, there were significant specific indirect effects from RPG quality to Burnout via self-efficacy, autonomy, and social support (i.e. the $95 \%$ confidence interval excluded zero). The findings indicate that the relationship between more effective RPGs and lower Burnout was completely mediated (explained) by self-efficacy, autonomy, and social support.

Secondary Traumatic Stress. The standardised coefficients depicting the relationship pathways between quality RPGs and Secondary Traumatic Stress are presented in Figure 3. The overall model explained significant variance in Secondary Traumatic Stress, $R^{2}=.18, F(6,177)=6.37, p<$. oo1. Total effect revealed that higher scores of RPG quality did not significantly predict lower Secondary Traumatic Stress scores. However, after accounting for the mediators, the direct effect was increased and revealed a significant positive relationship. The unexpected finding, that more effective RPGs significantly increased Secondary Traumatic Stress, should be interpreted cautiously due to the presence of suppression (i.e. direct > total effect). Higher scores on self-efficacy, 


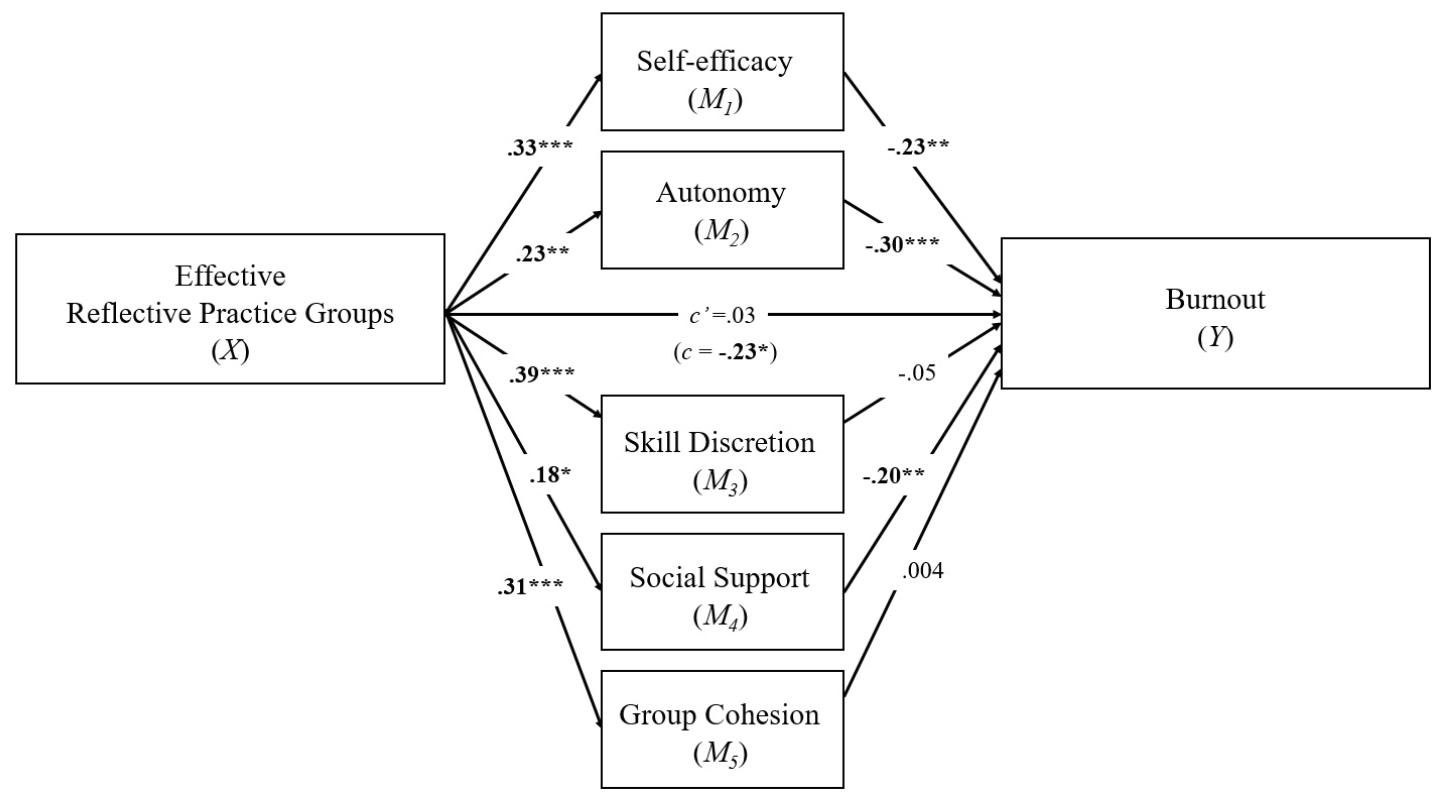

FIGURE 2: STANDARDISED REGRESSION COEFFICIENTS FOR THE PARALLEL MEDIATION MODEL TESTING THE INDIRECT RELATIONSHIP BETWEEN QUALITY REFLECTIVE PRACTICE GROUPS AND BURNOUT THROUGH THE MEDIATORS OF SELF-EFFICACY, AUTONOMY, SKILL DISCRETION, SOCIAL SUPPORT, AND GROUP COHESION $(N=184) . C=$ TOTAL EFFECT. $C^{\prime}=$ DIRECT EFFECT. ${ }^{\star} P<.05 ;{ }^{* \star} P<.01 ;{ }^{* \star *} P<.001$.

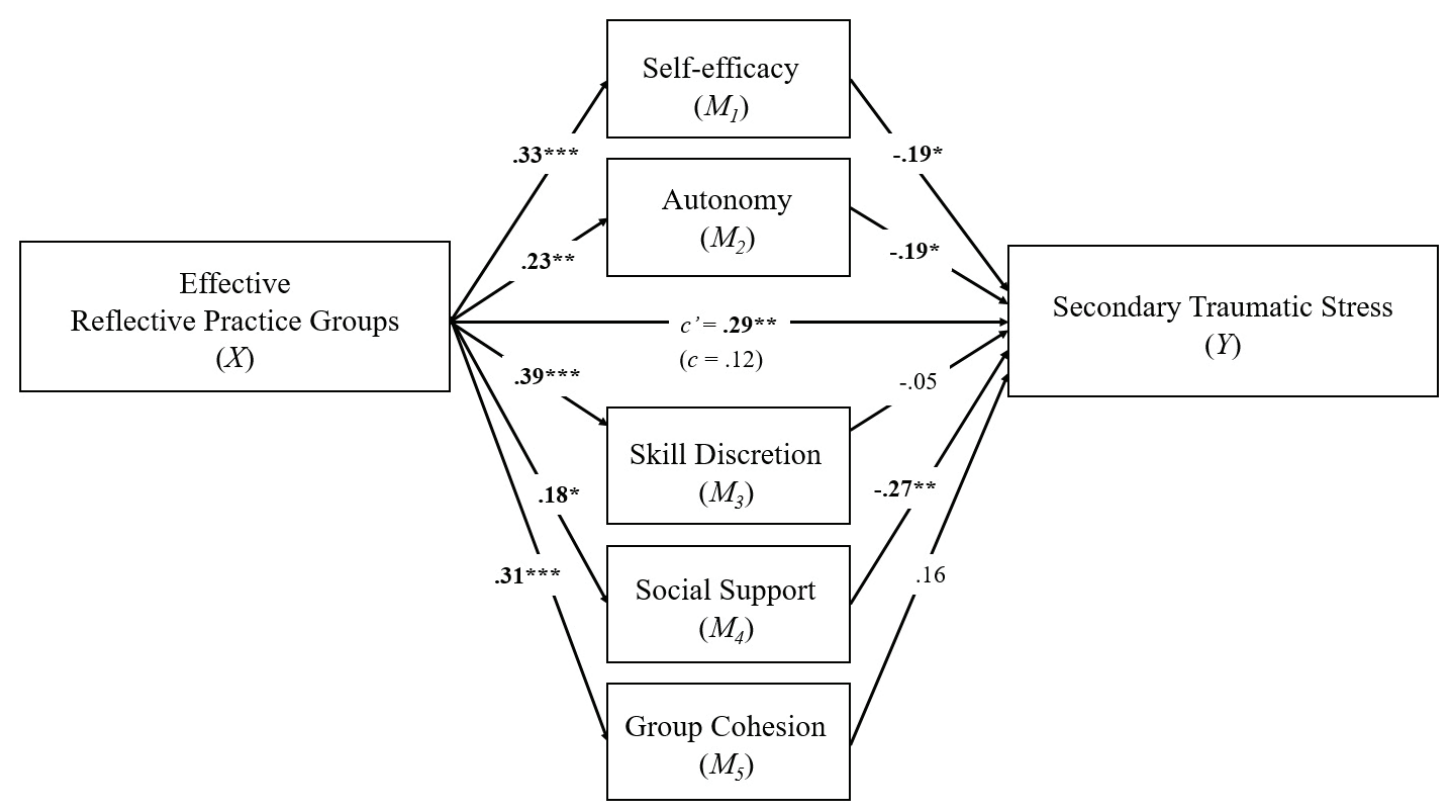

FIGURE 3: STANDARDISED REGRESSION COEFFICIENTS FOR THE PARALLEL MEDIATION MODEL TESTING THE INDIRECT RELATIONSHIP BETWEEN MORE EFFECTIVE REFLECTIVE PRACTICE GROUPS AND SECONDARY TRAUMATIC STRESS THROUGH THE MEDIATORS OF SELF-EFFICACY, AUTONOMY, SKILL DISCRETION, SOCIAL SUPPORT, AND GROUP COHESION $(N=184) . C=$ TOTAL EFFECT. $C^{\prime}=$ DIRECT EFFECT. ${ }^{\star} P<.05 ;{ }^{\star \star} P<.01 ;{ }^{* \star *} P<.001$.

autonomy, and social support significantly predicted lower Secondary Traumatic Stress scores. The relationship between group cohesion and Secondary Traumatic Stress was in the positive direction and approached significance $(p=.051)$.

As shown in Table 4, there were significant indirect effects from RPG quality to lower Secondary Traumatic Stress through self-efficacy, autonomy, and social support.
The specific indirect effect via group cohesion was also significant, although in a positive direction, indicating negative suppression (i.e. path and correlation in opposition directions) is present in the model and again should also be interpreted cautiously. Overall, these findings suggest the relationship between more effective RPGs and lower Secondary Traumatic Stress was partially mediated by selfefficacy, autonomy, and social support. 


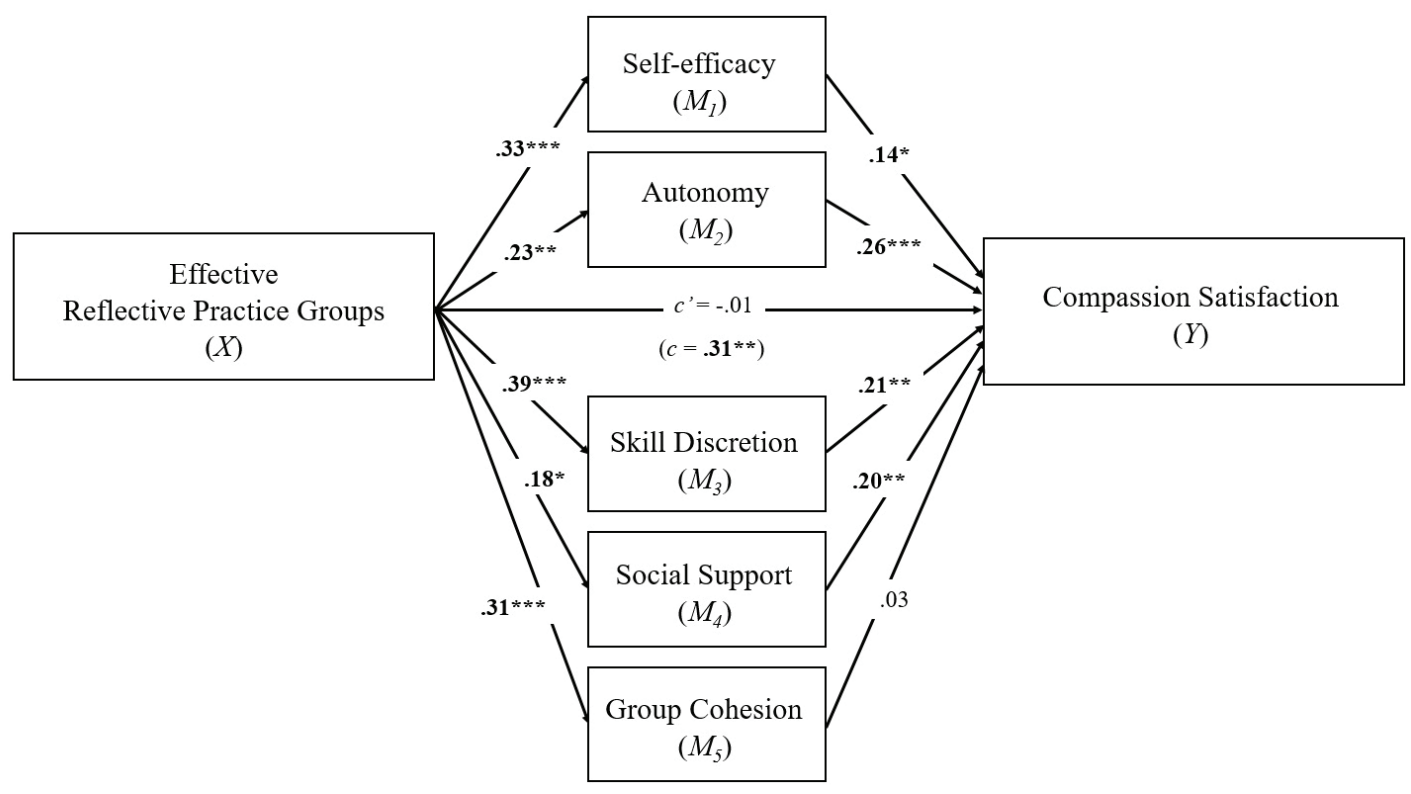

FIGURE 4: STANDARDISED REGRESSION COEFFICIENTS FOR THE PARALLEL MEDIATION MODEL TESTING THE INDIRECT RELATIONSHIP BETWEEN MORE EFFECTIVE REFLECTIVE PRACTICE GROUPS AND COMPASSION SATISFACTION THROUGH THE MEDIATORS OF SELFEFFICACY, AUTONOMY, SKILL DISCRETION, SOCIAL SUPPORT, AND GROUP COHESION $(N=184) . C^{\prime}=$ TOTAL EFFECT. $C^{\prime}=$ DIRECT $^{\prime}$ EFFECT. *P<.05; ${ }^{* *} P<.01 ;{ }^{* \star *} P<.001$.

Compassion Satisfaction. The standardised coefficients depicting the relationship pathways between quality RPGs and Compassion Satisfaction scores are presented in Figure 4. The overall model accounted for significant unique variation in Compassion Satisfaction scores, $R^{2}$ $=.29, F(6,177)=12.39, p<.001$. Total effect pathway revealed higher scores of RPG quality significantly predicted greater Compassion Satisfaction scores. Controlling for the mediators, the direct effect decreased and was rendered non-significant. Higher scores of self-efficacy, autonomy, skill discretion, and social support significantly predicted greater Compassion Satisfaction scores, whereas group cohesion did not significantly contribute. As shown by Table 4, there were significant specific indirect effects from RPG quality to Compassion Satisfaction via autonomy, skill discretion, and social support. These findings indicate that the relationship between more effective RPGs and greater Compassion Satisfaction was completely mediated by autonomy, skill discretion, and social support.

\section{DISCUSSION}

The present study aimed to extend previous research by exploring the relationship between the quality of RPGs (as determined by nurses who attend), personal and job resources, and ProQoL. Findings indicated that nurses who rated their RPGs more highly also scored higher for selfefficacy, autonomy, skill discretion, social support, and group cohesion. After considering the possible explanatory role of all resources in parallel, some appeared to be more closely implicated in the relationship between higher quality RPGs and greater ProQoL; specifically significantly lower burnout, secondary traumatic stress, and increased compassion satisfaction. Interestingly, the apparent link between each resource and the different ProQoL components varied; with autonomy and social support found to be the most constantly associated resources across all components, and group cohesion the least.

In terms of the first aim of this study, results revealed that nurses who attended what they perceived to be higher quality RPGs reported significantly greater resources of self-efficacy, autonomy, skill discretion, social support, and group cohesion. The data might suggest that nurses with higher personal and job resources appreciate RPGs and rate them more highly, however it could also indicate that nurses who perceive RPGs as beneficial are able to cultivate a range of resources. This would be consistent with the themes emerging from previous qualitative studies that indicate nurses perceive a range of benefits from RPG attendance including support, collaborative learning, job confidence and skills development. ${ }^{16,22,26,37-39}$ It is proposed that the findings of the study can be conceptualised through a job crafting lens. The RPG model proposes that groups be implemented in a way that engages and empowers the nursing work group. RPGs are voluntary and utilise a semi-structured, collaborative, process-focussed facilitation approach to create a sense of psychological safety and enhance reflection. The aim is to allow nurses to access benefits in a way that considers the specific needs of both the individual and the work group..$^{1,14,16,22,25}$ Gordon and colleagues, ${ }^{40}$ proposed 
that job crafting interventions allowed nurses to seek out resources; sourcing new opportunities for autonomy, social support, learning, and professional development. The present study contributes to job crafting literature by utilising the job demands-resources model as a framework to explore the relationship between quality RPGs, conceptualised as a job crafting exercise, and a range of personal and job resources.

The second aim of the study was to explore how the presence of various personal and job resources might be seen to mediate the relationship between more effective RPGs and greater ProQoL These findings will be discussed in order of relative value, as determined by the strength of the correlation between resources and higher nurse ProQoL. Autonomy and social support were found to be those most consistently linked to each of the ProQoL outcomes.

\section{AUTONOMY AND SOCIAL SUPPORT}

The current study indicates that autonomy and social support significantly mediate the relationship between higher quality RPGs and all components of enhanced ProQoL, including reduced burnout, reduced secondary traumatic stress, and increased compassion satisfaction. Once again, it might be that more resilient nurses with higher ProQoL value the groups more, however it is also possible that attending effective RPGs might have a positive impact on nurses' sense of control over certain aspects of their work, whilst cultivating a culture of support within the workplace. In addition, nurses experiencing less compassion fatigue may be better equipped to manage patient distress and derive a greater sense of fulfilment from their work.

The current study is the first to explore possible mechanisms for the relationship between RPGs and ProQoL of nurses. As such, there is a lack of research against which to directly compare present findings. Nonetheless, findings do appear to be broadly consistent with the job demands-resources model. For example, job crafting interventions have been found to increase autonomy and social support significantly and this has been associated with increased job performance, ${ }^{41}$ work engagement, and decreased negative affect. ${ }^{42}$

Previous research has indicated that the clinical discussions facilitated during RPGs allow nurses to gain new clinical insights and this may help them manage clinical issues more effectively. This might empower nurses to act more independently in their practice, thereby providing a sense of autonomy. ${ }^{43}$ This would be consistent with longitudinal studies of nurses that indicate autonomy significantly predicts lower burnout, ${ }^{44}$ increased job satisfaction, ${ }^{45}$ and work engagement. 33

Social support has also been found to reduce the level of distress associated with stressful experiences. ${ }^{46}$ Qualitative evidence has suggested that quality RPGs provide the opportunity for nurses to gain social support in several ways; emotional support (feeling accepted), informational support (guidance, advice) and social companionship. ${ }^{16,47}$ This notion aligns with the concept of job crafting within the job demands-resources framework, as RPGs enable nurses to source different forms of support to help mitigate workplace stress. ${ }^{16,22,37,39}$

Taken together, autonomy and social support would appear to be influential mechanisms in the relationship between more effective RPGs and increased ProQoL. It is still not possible to categorically determine cause and effect, but this correlation suggests that nurses could increase resilience by utilising their skillset and by sharing their concerns with supportive team members in RPGs.

\section{SELF-EFFICACY}

Findings from the current study revealed that self-efficacy significantly mediated the relationship between higher quality RPGs and two components of ProQoL: reduced burnout and secondary traumatic stress. Attending more effective RPGs may foster nurses' confidence in their ability to deliver effective care.

When considered within the job demands-resources model, this suggests that RPGs might serve to empower nurses to cultivate confidence in their ability to manage work stress, allow then to process emotional labour, and help them to feel more effective within their role. In accordance with Bandura, ${ }^{48}$ attending quality RPGs possibly fosters selfefficacy through a mixture of vicarious experiences, verbal persuasion from colleagues, and reflection on performance accomplishments. As self-efficacy grows, nurses may begin to perceive difficult tasks (e.g. work overload) as challenges, reducing feelings of stress, anxiety, and helplessness.

An unanticipated finding from the current study was that the indirect path, through self-efficacy, between the quality of RPGs and Compassion Satisfaction did approach, but was not quite, significant. Future research in larger samples may further clarify the relationships of influential factors for the compassion satisfaction component of ProQoL.

\section{SKILL DISCRETION}

Findings from the current study indicate that skill discretion mediates the relationship between higher quality RPGs and increased compassion satisfaction. This could be seen to indicate that effective RPGs might provide the opportunity for creativity and the learning of new skills. One possible explanation for this apparent relationship might be that nurses perceive themselves as being able to provide greater quality care due to the gaining of new clinical insights and skills in RPGs. A longitudinal study by Petrou and colleagues, ${ }^{49}$ found participants who attended a job crafting intervention reported significantly greater skill discretion and work engagement 12-months later. 
In contrast, skill discretion did not appear to mediate the relationship between quality RPGs and reduced burnout or secondary traumatic stress. That is, the perception that they were gaining clinical insights and abilities through RPGs did not seem to affect nurses perceived effectiveness within their role nor protect them from being affected by their patients' suffering. This contrasts with earlier research, which found lower skill discretion in human service workers was associated with greater burnout..$^{\circ}$ This disparity could be explained by the different job demands of hospital-based nursing, such as high workloads, long hours, and time pressures. It is conceivable that under such conditions, applying new skills may be more difficult.

\section{GROUP COHESION}

Unexpectedly, and in contrast to the job demandsresources model, group cohesion did not appear to mediate relationships between quality RPGs and increased ProQoL. Although quality RPGs did appear to increase feelings of trust, acceptance, and empathy for fellow nurses, this did not necessarily translate into an increased sense of meaning or role effectiveness. These results differ from previously published nursing studies that found group cohesion was associated with significantly greater ProQoL and greater job satisfaction. ${ }^{51,52}$ This may be explained by the fact that these other studies may not have explored the relationship between group cohesion and ProQoL in the presence of other job resources.

Interestingly, the current study also revealed that increased group cohesion was associated with greater secondary traumatic stress in the mediation model, but not the correlations. This finding, along with the direct effect of RPGs being greater than the total effect, indicates suppression. The results for these variables should be interpreted with caution as group cohesion and social support were strongly and positively correlated. It could be speculated that nurses who attend RPGs might experience vicarious trauma when distressing clinical cases are discussed and colleague's reactions to traumatic experiences are explored, eliciting identification and empathetic interpersonal connections between colleagues. Additional research is needed to elucidate the complex relationships between group cohesion, social support, and secondary traumatic stress and the experience of working through traumatic work events within RPGs.

Collectively, the findings from the present study indicate that the relationship between effective RPGs and greater ProQoL may be mediated by several key personal and job resources. Whilst cause and effect are still not clear, and the link may be that nurses with higher levels of personal resources appreciate RPGs more, the study does support previous research that indicates the benefits of quality RPGs might stem from the provision of a trustworthy, supportive and constructive space in which nurses can actively develop these important resources, ${ }^{16,22,39}$ positively impacting ProQoL. ${ }^{1}$ Given that specific resources seem to have differing effects on each component of ProQoL, a growing body of evidence is beginning to identify the potential of RPGs in providing a valuable and practical intervention that provides nurses the opportunity to access a range of benefits that align with, and address, their individual needs and the needs of the workgroup..$^{6,22,37}$

\section{LIMITATIONS AND FUTURE RESEARCH DIRECTIONS}

This study utilised a cross-sectional design to test an assumed causal relationship and results should be interpreted with care. Whilst there is strong theoretical support for the proposed relationship, it is imperative that future studies examine causality longitudinally. This is important as it is possible that individuals with greater ProQoL are more likely to attend RPGs and perceive the groups more positively. Although beyond the scope of this study, it is also possible that relationships between RPG quality and ProQoL are moderated by individual or environmental factors (e.g. personality, number of RPGs attended, and managerial support of RPGs). Future studies may benefit from investigating the moderating role of such factors. It is also recommended future studies clarify the complex relationship between RPGs, group cohesion, and secondary traumatic stress. Despite these limitations, the current study contributes to the nursing literature by providing a theoretically driven model to explain how attending effective RPGs increases nurses ProQoL.

\section{CONCLUSION}

The current study is the first to explore the mediating mechanisms in which the job crafting experience of quality RPGs might influence nurses ProQoL. Results indicate that effective RPGs may increase nurses' personal and job resources of self-efficacy, autonomy, skill discretion, social support, and group cohesion. These findings pose some theoretical implications within the framework of the job demands-resource model and the function of job crafting. That is, that voluntary attendance at quality RPGs may allow nurses to proactively develop strategies and resources that buffer against workplace demands, thereby mitigating the negative effects of ProQoL and supporting compassion satisfaction.

Overall, the findings provide further indications that quality RPGs $s^{15,16}$ are an effective clinical supervision intervention that could allow healthcare organisations to support and empower nurses and help them deal with the challenges of their profession. 


\section{RELEVANCE FOR CLINICAL PRACTICE}

Based on the results of this study, it is important to ensure RPGs are of high quality and effective. ${ }^{15,16}$ By actively identifying signs that members are seeking social support, facilitators can help other group members recognise subliminal invitations and model effective ways of providing such support. When considering RPGs within a job crafting perspective, facilitators need to strike a balance between offering guidance and providing nurses freedom to direct discussions. Based on current findings, it seems beneficial that RPGs be delivered in an unstructured or semi-structured group format with voluntary attendance and no managerial coercion. Empowering nurses to be active agents in the RPG process enhances the formative and supportive aspects of the groups, whilst providing an opportunity to positively impact nursing ProQoL.

Acknowledgements: The authors wish to acknowledge and thank Mr Andrew Zanos and Ms Phoebe Rattray for their assistance with data collection during the recruitment phase of this study, in addition to all of the nurses who participated. This study was supported by Sunshine Coast Hospital and Health Services.

Disclosure: This research did not receive any financial support and no specific grants were provided from funding agencies in the public, commercial, or not-for-profit sectors. There are no conflicts of interest regarding this publication.

Conflict of Interest: CD maintains a private supervision practice under the name 'Reflective Practice Group’.

\section{REFERENCES}

1. Davey BR, Byrne SJ, Millear PM, Dawber C, Medoro L. Evaluating the impact of reflective practice groups for nurses in an acute hospital setting. Aust J Adv Nurs. 2021;38(1):6-17.

2. Stamm B. The concise manual for the professional quality of life scale. ProQOL. 2010. Available from: https://proqol.org/proqol-manual

3. Lee E, Daugherty J, Eskierka K, Hamelin K. Compassion fatigue and burnout: one institution's interventions. J Perianesth Nurse. 2019;34(4):767-73.

4. Sung K, Seo Y, Kim JH. Relationships between compassion fatigue, burnout, and turnover intention in Korean hospital nurses. J Korean Acad Nurs. 2012;42(7):1087-94.

5. Aggar C, Gordon CJ, Thomas THT, Wadsworth L, Bloomfield J. Evaluation of a community transition to professional practice program for graduate registered nurses in Australia. Nurse Educ Prac. 2018;32:101-7.

6. Australia HW. Australia's Future Health Workforce - Nurses Overview Report. 2014. Available from: https://www. health.gov.au/resources/publications/nurses-australiasfuture-health-workforce-reports?utm source=health.gov. au\&utm medium=callout-auto-custom\&utm campaign=digital transformation

7. Maslach C, Jackson SE. The measurement of experienced burnout. J Organ Behav. 1981;2(2):99-113.
8. Bakker AB, Demerouti E. The job demands-resources model: state of the art. J Manag Psych. 2007;22(3):309-28.

9. Bakker AB, Demerouti E, Sanz-Vergel Al. Burnout and work engagement: The JD-R approach. Ann Rev Organ Psychol Organ Behav. 2014;1(1):389-411.

10. Jourdain $G$, Chenevert D. Job demands-resources, burnout and intention to leave the nursing profession: A questionnaire survey. Int J Nurs Stud. 2010;47(6):709-22.

11. Barbier M, Dardenne B, Hansez I. A longitudinal test of the Job Demands-Resources model using perceived stigma and social identity. Eur J Work Organ Psychol. 2013;22(5):532-46.

12. Alarcon GM. A meta-analysis of burnout with job demands, resources, and attitudes. J Vocat Behav. 2011;79(2):549-62

13. McVicar A. Scoping the common antecedents of job stress and job satisfaction for nurses (2000-2013) using the job demandsresources model of stress. J Nurs Manag. 2016;24(2):E112-36.

14. Dawber C. Reflective practice groups for nurses: a consultation liaison psychiatry nursing initiative: part 1 - The model. Int J Ment Health Nurs 2013;22(2):135-44.

15. Kurtz A. How to run reflective practice groups: a guide for healthcare professionals. Routledge; 2019.

16. Reschke DJ, Dawber C, Millear PM, Medoro LJA. Group clinical supervision for nurses: process, group cohesion and facilitator effect. Aust J Adv Nurs. 2021;38(3):66-74.

17. Dawber C, O'Brien T. A longitudinal, comparative evaluation of reflective practice groups for nurses working in intensive care and oncology. J Nurs Care. 2013;2(3):138.

18. Tims M, Bakker AB. Job crafting: towards a new model of individual job redesign. J Ind Psych. 2010;36(2).

19. Demerouti E. Design your own job through job crafting. Eur Psychol. 2014;19(4):237-47.

20. Tims $M$, Bakker $A B$, Derks $D$. The impact of job crafting on job demands, job resources, and well-being. J Occup Health Psychol. 2013;18(2):230-40.

21. Horton S, Drachler ML, Fuller A, Leite JCC. Development and preliminary validation of a measure for assessing staff perspectives on the quality of clinical group supervision. Int J Lang Commun Disord. 2008;43(2):126-34.

22. Dawber C. Reflective practice groups for nurses: a consultation liaison psychiatry nursing initiative: part 2 - the evaluation. Int J Ment Health Nurs 2013;22(3):241-8.

23. Hyrkas K. Clinical supervision, burnout, and job satisfaction among mental health and psychiatric nurses in Finland. Issues Ment Health Nurs. 2005;26(5):531-56.

24. Gonge H, Buus N. Model for investigating the benefits of clinical supervision in psychiatric nursing: a survey study. Int J Ment Health Nurs. 2011;20(2):102-11.

25. Koivu A, Saarinen Pl, Hyrkas K. Who benefits from clinical supervision and how? The association between clinical supervision and the work-related well-being of female hospital nurses. J Clin Nurs. 2012;21(17-18):2567-78.

26. Fenton K, Kidd K. Reflective practice groups in a mental health inpatient setting. Ment Health Prac. 2019;22(3):28-33.

27. Wallbank S, Hatton S. Reducing burnout and stress: the effectiveness of clinical supervision. Community Pract. 2011;84(7):31-5

28. Proctor B, Marken M, Payne M. Enabling and ensuring Supervision: A co-operative exercise in accountability. Leicester, UK: Leicester National Youth Bureau and Council for Education and Training in Youth and Community Work; 1988. 
29. Gabrielsson S, Engström Å, Gustafsson S. Evaluating reflective practice groups in a mental health context: Swedish translation and psychometric evaluation of the clinical supervision evaluation questionnaire. BMC nurs. 2019;18(2)

30. Jerusalem M, Schwarzer R. The general self-efficacy scale. 1979.

31. Voydanoff P. The effects of work demands and resources on work-to-family conflict and facilitation. J Marriage Fam. 2004;2(66):398-412.

32. Schwartz JE, Pieper CF, Karasek RA. A procedure for linking psychosocial job characteristics data to health surveys. Am J Pub Health. 1988;78(8):904-9.

33. van der Ploeg E, Kleber RJ. Acute and chronic job stressors among ambulance personnel: predictors of health symptoms Occup Environ Med. 2003;60:40-6.

34. Wongpakaran T, Wongpakaran N, Intachote-Sakamoto R, Boripuntakul T. The Group Cohesiveness Scale (GCS) for psychiatric inpatients. Perspect Psychiatr Care. 2013;49(1):58-64.

35. Hayes AF. Introduction to mediation, moderation, and conditional process analysis: a regression-based approach. New York: The Guilford Press; 2013.

36. Fritz MS, Mackinnon DP. Required sample size to detect the mediated effect. Psychol Sci. 2007;18(3):233-9.

37. McVey J, Jones T. Assessing the value of facilitated reflective practice groups. Cancer Nurs Prac. 2012;11(8):32-7.

38. Nordbøe C, Enmarker I. The benefits of person-centred clinical supervision in municipal healthcare- Employees' experience. Open J Nurs. 2017;7(5):548-60.

39. O'Neill L, Johnson J, Mandela R. Reflective practice groups: are they useful for liaison psychiatry nurses working within the emergency department? Arch Psychiatr Nurs. 2019;33(1):85-92

40. Gordon HJ, Demerouti E, Le Blanc PM, Bakker AB, Bipp T, Verhagen MAMT. Individual job redesign: job crafting interventions in healthcare. J Vocat Behav. 2018;104:98-114.

41. van Wingerden J, Bakker AB, Derks D. The longitudinal impact of a job crafting intervention. Eur J Work Organ Psychol. 2016;26(1):107-19

42. van den Heuvel M, Demerouti E, Peeters MCW. The job crafting intervention: effects on job resources, self-efficacy, and affective well-being. J Occup Organ Psychol. 2015;88(3):511-32.

43. Deci EL, Ryan RM. Conceptualizations of intrinsic motivation and self-determination. Intrinsic Motivation \& SelfDetermination in Human Behavior. New York: Plenum; 1985; $11-40$.

44. Adriaenssens J, De Gucht V, Maes S. Causes and consequences of occupational stress in emergency nurses, a longitudinal study. J Nurs Manag. 2013;23(3):346-58.

45. Jonsson S. Psychosocial work environment and prediction of job satisfaction among Swedish registered nurses and physicians a follow-up study. Scand J Caring Sci. 2012;26(2):236-44.

46. Li L, Ruan H, Yuan WJ. The relationship between social support and burnout among ICU nurses in Shanghai: a cross-sectional study. Chinese Nurs Res. 2015;2(2-3):45-50.

47. Cross W, Moore A, Ockerby S. Clinical supervision of general nurses in a busy medical ward of a teaching hospital. Contemp Nurse. 2010;35(2):245-53.

48. Bandura A. Self-efficacy: toward a unifying theory of behavioral change. Psychol Rev. 1977;84(2):191-215.
49. Petrou P, Demerouti E, Peeters MCW, Schaufeli WB, Hetland J. Crafting a job on a daily basis: Contextual correlates and the link to work engagement. J Organ Behav. 2012;33(8):1120-41.

50. Rafferty Y, Friend R, Landsbergis PA. The association between job skill discretion, decision authority and burnout. Work Stress. 2001;15(1):73-85

51. Li A, Early SF, Mahrer NE, Klaristenfeld JL, Gold JI. Group cohesion and organizational commitment: protective factors for nurse residents' job satisfaction, compassion fatigue, compassion satisfaction, and burnout. J Prof Nurs. 2014;30(1):89-99

52. Kalisch BJ, Lee H, Rochman M. Nursing staff teamwork and job satisfaction. J Nurs Manag. 2010;18(8):938-47. 